九州大学学術情報リポジトリ

Kyushu University Institutional Repository

\title{
ON A MAXIMIZATION PROBLEM ARISING FROM A POSITIVE RECIPROCAL MATRIX IN AHP
}

Shiraishi, Shunsuke

Faculty of Economics, Toyama University

Obata, Tsuneshi

Faculty of Engineering, Oita University

https://doi.org/10.5109/13511

出版情報: Bulletin of informatics and cybernetics. 34 (2), pp.91-96，2002-12. Research Association of Statistical Sciences

バージョン :

権利関係 : 


\title{
ON A MAXIMIZATION PROBLEM ARISING FROM A POSITIVE RECIPROCAL MATRIX IN AHP
}

\author{
By \\ Shunsuke ShIraISBi* and Tsuneshi Obama ${ }^{\dagger}$
}

\begin{abstract}
The maximization of the third coefficient of the characteristic polynomial has been proved useful. It has a posynomial form as a function of entries of a positive reciprocal matrix. Hence one can transform it to a convex function. We will prove the existence of a solution of the minimization problem of the resulting convex function.
\end{abstract}

Key Words and Phrases: Analytic Hierarchy Process, Coefficient of characteristic polynomial, Positive reciprocal matrix, Posynomial programming, Principal eigenvalue.

\section{Introduction and Preliminaries}

The pairwise comparison procedure is the heart of AHP(Analytic Hierarchy Process) and it gives a positive reciprocal matrix of the following form:

$$
A=\left(\begin{array}{cccc}
1 & a_{12} & \cdots & a_{1 n} \\
1 / a_{12} & 1 & \cdots & a_{2 n} \\
\vdots & \vdots & \ddots & \vdots \\
1 / a_{1 n} & 1 / a_{2 n} & \cdots & 1
\end{array}\right)
$$

where $a_{i j}>0$ for all $i, j=1, \ldots, n$ (Golden et al., 1989, Saaty, 1980, 1994, Tone, 1986). The principal eigenvalue $\lambda_{\max }$ of $A$ plays an important role as a consistency index C.I. which measures inconsistency of the pairwise comparison. It is defined as follows

$$
\text { C.I. }=\frac{\lambda_{\max }-n}{n-1} \text {. }
$$

Decision makers are required to do pairwise comparisons so that C.I. would be as small as possible. Needless to say that $\lambda_{\max }$ is a maximal solution of the characteristic polynomial of $A$ which is denoted by

$$
P_{A}(\lambda):=\operatorname{det}(\lambda E-A)=\lambda^{n}+c_{1} \lambda^{n-1}+\cdots+c_{n-1} \lambda+c_{n} .
$$

* Faculty of Economics, Toyama University Toyama 930-8555 Japan. shira@eco.toyama-u.ac.jp Supported in part by Grant-in-Aid for Scientific Research (B) No.14340037 from Japan Society for the Promotion of Science.

t Faculty of Engineering, Oita University Oita 870-1192 Japan. obata.9csis.oita-u.ac.jp 
In our prior work (Obata et al., 1999), we verified the strong relationship between the third coefficient $c_{3}$ and C.I. by a computational experiment. We observed that C.I. gets smaller as $c_{3}$ gets greater.

One may wonder that one can calculate the value of $c_{3}$ easily. With the aid of the following simple form of the third coefficient ${ }^{1}$, one will agree that the calculation is easy.

Proposition 1.1. (Shimaishi et al., 1998) Let $n \geq 3$. If $A$ is a pasitive reciprocal $n \times n$ matrix, then

$$
\begin{aligned}
c_{3} & =\sum_{i<j<k}\left\{2-\left(\frac{a_{i j} a_{j k}}{a_{i k}}+\frac{a_{i k}}{a_{i j} a_{j k}}\right)\right\} \\
& =2\left(\begin{array}{l}
n \\
3
\end{array}\right)-\sum_{i<j<k}\left(\frac{a_{i j} a_{j k}}{a_{i k}}+\frac{a_{i k}}{a_{i j} a_{j k}}\right),
\end{aligned}
$$

so that $c_{3} \leq 0$.

On the other hand, decision makers often make an incomplete matrix of the form ${ }^{2}$ :

$$
A(w)=\left(\begin{array}{cccccc}
1 & a_{12} & \cdots & w_{1} & \cdots & a_{1 n} \\
1 / a_{12} & 1 & \cdots & \cdots & \cdots & a_{2 n} \\
\vdots & \vdots & \ddots & & w_{m} & \vdots \\
1 / w_{1} & \vdots & & \ddots & & \vdots \\
\vdots & \vdots & 1 / w_{m} & & \ddots & \vdots \\
1 / a_{1 n} & 1 / a_{2 n} & \cdots & \cdots & \cdots & 1
\end{array}\right),
$$

where $w=\left(w_{1}, \ldots, w_{m}\right)$ denotes the missing elements. Since C.I. is required to be as small as possible, it is natural to find a solution $w>0$ of the following minimization problem:

$$
\min _{w>0} \lambda_{\max }(w)
$$

where $\lambda_{\max }(w)$ denotes the principal eigenvalue of $A(w)$. However this problem is hard to solve exactly. By the way, we saw that C.I. (hence $\lambda_{\max }$ ) gets smaller as $c_{3}$ gets greater. So it is natural to propose the following maximization problem as a heuristic method which is alternate of (1.2) (Shiraishi et al., 1998) :

$$
\max _{w>0} c_{3}(w)
$$

where $c_{3}(w)$ denotes the third coefficient of the characteristic polynomial of $A(w)$.

In this paper, first we will show that the problem (1.3) can be converted to an equivalent convex minimization problem. Next, we will show that it always has an optimal solution (existence of an optimal solution).

\footnotetext{
${ }^{1}$ From the general theory of the characteristic polynomials, we know that $c_{1}=-n$. We can also show that $c_{2}=0$ (see Shiraishi et al., 1998).

2 e.g. unwillingmess to make a direct comparison between two alternatives, being unsure of some of the comparison, etc. (see Harker, 1987).
} 


\section{Equivalent Convex Programming and Existence of Solutions}

The maximization problem (1.3) is equivalent to the minimization of $-c_{3}(w)$. Since the main part of $c_{3}(w)$ is written in the simple form (1.1), (1.3) turns to be the following problem

$$
\min _{w>0} \sum_{i<j<k}\left\{\frac{a_{i j} a_{j k}}{a_{i k}}+\frac{a_{i k}}{a_{i j} a_{j k}}\right\}
$$

in which some $a_{i j}$ 's are regarded as the variables $w_{1}, \ldots, w_{m}{ }^{3}$. The function (2.1) is of the form of posynomials. Posynomials can be converted to convex functions by using the exponential transformation (see Ben-Israel et al., 1981, Fukushima, 1980). By setting $b_{i j}=\log a_{i j}$, we have

$$
\min \sum_{i<j<k}\left(\exp \left(b_{i j}+b_{j k}-b_{i k}\right)+\exp \left(-b_{i j}-b_{j k}+b_{i k}\right)\right) .
$$

Since $\log$ is monotone, the problem (2.1) is equivalent to:

$$
\min \log \left\{\sum_{i<j<k}\left(\exp \left(b_{i j}+b_{j k}-b_{i k}\right)+\exp \left(-b_{i j}-b_{j k}+b_{i k}\right)\right)\right\} .
$$

The variable vector $w=\left(w_{1}, \ldots, w_{m}\right)$ is also transformed to $t=\left(t_{1}, \ldots, t_{m}\right)$ by setting $t_{l}=\log w_{l}$. Hence the formula (2.2) includes terms as follows:

$$
\exp \left(t_{l}+b_{j k}-b_{i k}\right), \quad \exp \left(t_{l_{1}}+b_{j k}-t_{l_{2}}\right), \cdots, \text { etc. }
$$

Let $f(x)=f\left(x_{1}, \cdots, x_{K}\right)$ be a convex function defined by

$$
f\left(x_{1}, \cdots, x_{K}\right):=\log \left(e^{x_{1}}+\cdots+e^{x_{K}}+e^{-x_{1}}+\cdots+e^{-x_{K}}\right),
$$

where $K=\left(\begin{array}{l}n \\ 3\end{array}\right)$. Then (2.3) implies that there exist a matrix $B$ and a vector $b$ such that $x=B t+b$. Hence it suffices to solve the following unconstrained convex minimization problem:

$$
\min _{t \in R^{m}} f(B t+b)
$$

In the remaining part of this section, we will prove the existence of an optimal solution of the problem (2.4). For this purpose, we recall the definition of the recession function of a convex function and its properties (see Rockafellar, 1970).

Definition 2.1. Let $g: R^{N} \rightarrow R$ be a continuous convex function. Then, for $x, d \in R^{N}$, the recession function of $g$ is defined by

$$
g_{\infty}(d):=\sup _{\lambda>0} \frac{g(x+\lambda d)-g(x)}{\lambda}=\lim _{\lambda \rightarrow \infty} \frac{g(x+\lambda d)-g(x)}{\lambda} .
$$

We note that the value of the recession function is independent to the choice of $x$.

\footnotetext{
$3 \mathrm{~m}$ is regarded as the number of the missing data, which we do not state precisely how many is it. It is an interesting problem to question how many missing data items are tolerated. This question was developed by Takeda et al. (1995).
} 
LEMMA 2.2. (Rockafellar, 1970) Let $g: R^{N} \rightarrow R$ be a continuous convex function and $L$ a linear subspace of $R^{N}$. If $g_{\infty}(d)>0$ for all $0 \neq d \in L$, then $\left.g\right|_{L}$ attains $a$ minimum, where $\left.g\right|_{L}$ denotes the restriction of $g$ on $L$.

Consider the composite function $h(x)=g(B x+b)$, where $B x+b$ is an affine transformation. The following assertion is immediate from the definition of the recession function.

LEMMA 2.3. It holds that

$$
h_{\infty}(d)=g_{\infty}(B d) \text {. }
$$

Let us consider the direct sum decomposition $R^{N}=(\operatorname{ker} B) \oplus(\operatorname{ker} B)^{\perp}$.

LEMMA 2.4. (Shiraishi, 1991) If $g_{\infty}(d)>0$ for all $d \neq 0$, then $h$ attains its minimum on $(\operatorname{ker} B)^{\perp}$. have

Proof. For any $0 \neq d \in(\operatorname{ker} B)^{\perp}$, we have $B d \neq 0$. Then, by Lemma 2.3 , we

$$
h_{\infty}(d)=g_{\infty}(B d)>0 .
$$

The conclusion follows from Lemma 2.2 .

Proposition 2.5. If $g_{\infty}(d)>0$ for all $d \neq 0$, then $h$ attains its minimum on $R^{N}$.

Proof. By Lemma 2.4, there exists a vector $\bar{x}$ such that

$$
h(x) \geq h(\bar{x}) \text { for all } x \in(\operatorname{ker} B)^{\perp} .
$$

For any $x \in R^{N}$, we decompose $x$ into $x=x_{1}+x_{2} \in(\operatorname{ker} B) \oplus(\operatorname{ker} B)^{\perp}$. Then we have

$$
\begin{aligned}
h(x) & =h\left(x_{1}+x_{2}\right)=g\left(B x_{1}+B x_{2}+b\right)=g\left(B x_{2}+b\right) \\
& =h\left(x_{2}\right) \geq h(\bar{x}) .
\end{aligned}
$$

This completes the proof.

We now apply this proposition to the Problem (2.4).

LEMMA 2.6. For any $0 \neq d \in R^{K}$, we have

$$
f_{\infty}(d)>0 \text {. }
$$

Proof. Since $\log \left(e^{y_{1}}+\cdots+e^{y_{2} K}\right) \geq \log e^{y_{k}}=y_{k}$ for all $k=1, \ldots, 2 K$, we have

$$
\begin{aligned}
f\left(x_{1}, \cdots, x_{K}\right) & =\log \left(e^{x_{1}}+\cdots+e^{x_{K}}+e^{-x_{1}}+\cdots+e^{-x_{K}}\right) \\
& \geq\left|x_{k}\right|,
\end{aligned}
$$

for all $k=1, \ldots, K$. Hence it holds that

$$
\begin{aligned}
f_{\infty}(d) & =\lim _{\lambda \rightarrow \infty} \frac{f(\lambda d)-f(0)}{\lambda} \\
& \geq \lim _{\lambda \rightarrow \infty} \frac{\lambda\left|d_{k}\right|}{\lambda}=\left|d_{k}\right|,
\end{aligned}
$$

for all $k=1, \ldots, K$. This implies that $f_{\infty}(d)>0$ for all $d \neq 0$.

THEOREM 2.7. The problem (2.4) always has an optimal solution.

Proof. It is clear from Proposition 2.5 and Lemma 2.6. 


\section{Conclusion}

In this paper, we presented the existence of a solution of Problem (1.3). It is easy to see that this problem sometimes has infinitely many solutions. So the new question arises. Which solution is the best candidate?

It is an answer to this question to introduce another measurement function and consider its optimization problem on the solution set of Problem (1.3). For example, Yamada et al. (1997) proposed such a function. However they did not touch upon existence of the solution. Since we showed that the solution set is non-empty, this problem is always feasible. Hence one may consider that the present paper gives theoretical foundations for this problem.

\section{Acknowledgement}

This paper is dedicated to Prof. Nobuyuki Nakajima of Toyama University for his 60 th birthday.

\section{References}

Ben-Israel, A., Ben-Tal, A. and Zlobec, S. (1981). Optimality in Nonlinear Pragramming: A Feasible Directions Approach, John Wiley, New York.

Fukushima, M. (1980). Theory of Nonlinear Optimization (in Japanese), Sangyo Tosho, Inc., Tokyo.

Golden, B.L., Wasil, E.A. and Harker, P.T. (Eds.) (1989). The Analytic Hieranchy Process, Springer Verlag, New York.

Harker, P.T. (1987). Alternative Modes of Questioning in the Analytic Hierarchy Process, Mathl. Modelling, 9, 353-360.

Obata, T., Daigo, M. and Shiraishi, S. (1998). A Computational Experiment on the Method for Estimating Priority Weights for Incomplete Comparison Matrices (in Japanese), Reports of the Faculty of Engineering Oita University, 37, 31-36.

Obata, T., Shiraishi, S., Daigo, M. and Nakajima, N. (1999). Assessment for an Incomplete Comparison Matrix and Improvement of an Inconsistent Comparison: Computational Experiments, Proceedings of The Fifth International Symposium on The Analytic Hierarchy Process, 200-205.

Rockafellar, R.T. (1970). Convex Analysis, Princeton University Press, Princeton.

Saaty, T.L. (1980). The Analytic Hierarchy Process, McGraw-Hill, New York.

Saaty, T.L. (1994). The Analytic Hierarchy Process Series VI, RWS Publication, Pittsburgh.

Shiraishi, S. (1991). $\varepsilon$-Directional Derivative of a Marginal Function in Parametrized Convex Programming, Bulletin of Informatics and Cybernetics, 24, 177-183.

Shiraishi, S., Obata, T. and Daigo, M. (1998). Properties of a Positive Reciprocal Matrix and Their Application to AHP, Journal of Operations Research Society of Japan, 41-3, 404-414. 
Takeda, E. and Yu, P.L. (1995). Assessing Priority Weights from Subsets of Pairwise Comparisons in Multiple Criteria Optimization Problems, European J. Oper. Res., 86, 315-331.

Tone, K. (1986). The Analytic Hiemarchy Process: Decision Making (in Japanese), Japanese Science and Technology Press, Tokyo.

Yamada, Y., Sugiyama, M. and Yamaki, N. (1997). Group Analytic Hierarchy Process Based on Consensus Making Model (in Japanese), Journal of Operations Research Society of Japan, 40-2, 236-244.

Received March 23, 2001

Revised July 24, 2002

Re-revised October 16, 2002 